\title{
On the FEL gain limit
}

\author{
V.N. Litvinenko
}

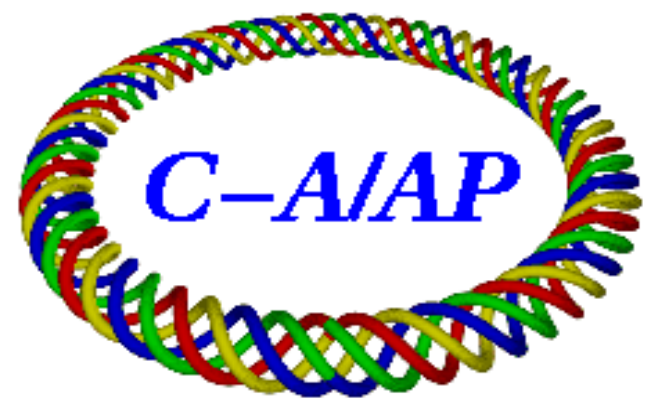

\section{Collider-Accelerator Department Brookhaven National Laboratory Upton, NY 11973}

Notice: This document has been authorized by employees of Brookhaven Science Associates, LLC under Contract No. DE-AC02-98CH10886 with the U.S. Department of Energy. The United States Government retains a nonexclusive, paid-up, irrevocable, world-wide license to publish or reproduce the published form of this document, or allow others to do so, for United States Government purposes. 
On the FEL gain limit

Vladimir N. Litvinenko

\section{Abstract}

This focus of this note is to determine what is the limitation on the amplification of the density modulation in FEL-type process caused by saturation. This derivation is applicable to any directional instability in the electron beam with a resonant wavenumber $k_{o} \equiv 2 \pi / \lambda_{0}$.

It is well known fact that e-beam instabilities, including that in FEL, are described by a set of self-consistent Maxwell and Vlasov equation. In classical limit, Maxwell equations are completely linear. The later is not true about the Vlasov equation. Hence, the later is responsible for the saturation (if any).

It is also a well established fact that Vlasov equation can be linearized when the density modulation is significantly smaller compared with initial beam density. In other words, the Vlasov equation becomes nonlinear (which can cause a saturation) when the density modulation becomes comparable with the initial beam density: $|\delta n| \sim n_{0}$.

When $\left|\delta n / n_{0}\right|<<1$ we can use linearized Vlassov equation, which then can be represented by a Green function. Here, for compactness, I consider a directional instability, where the response of the system on a perturbation can be described by onedimensional Green function. This is usually true for an FEL, where diffraction makes transverse dependences smooth compared with the fast longitudinal oscillations.

Here I also consider a response of the system to be much shorter compared with the electron bunch length, e.g. the e-beam density could be considered locally constant.

\section{General case}

In a linear approximation, the reaction of an FEL with constant electron beam density $n_{\circ}$ on a local perturbation

$$
\delta n=\delta\left(z-z_{o}\right)
$$

can be approximately described by a $1 \mathrm{D}$ Green function

$$
n(\tau)=n_{\circ}+\delta\left(z-z_{0}\right)+G_{\tau}\left(z-z_{0}\right),
$$

with obvious $\int G_{\tau}(z) d z=0$.

Let's consider a case of typical random start of SASE FEL with initial distribution of electrons as

$$
n_{o}(0, z)=\sum_{i=1}^{N} \delta\left(z-z_{i}\right) .
$$

where $\mathrm{N}$ is the number of electrons in the beam. Than the linear amplified FEL response reads:

$$
n_{\circ}(\tau, z)=+\sum_{i=1}^{N} \delta\left(z-z_{i}\right)+\sum_{i=1}^{N} G_{\tau}\left(z-z_{i}\right) .
$$


In the presence of density modulation imprinted by ions with charge $\mathrm{Z}$ and the effective density $\mathrm{X}=\mathrm{x} * \mathrm{Z}, \mathrm{x} \sim 1^{1}$

$$
n_{o}(\tau, z)=+\sum_{i=1}^{N_{e}} \delta\left(z-z_{i}\right)+\sum_{i=1}^{N_{e}} G_{\tau}\left(z-z_{i}\right)+X \sum_{j=1}^{N_{i}} G_{\tau}\left(z-z_{j}\right) .
$$

Let's calculate bunching factor, corresponding to the relative local density modulation by picking an arbitrary wavelet:

$$
\begin{aligned}
& b(\tau)=\frac{\int_{0}^{1} n(\tau, z) e^{i k_{0} z} d z}{\int_{0}^{1} n(\tau, z) d z}=\frac{\sum_{i, z_{i} \in\left\{0, \lambda_{0}\right\}} e^{i k_{0} z_{i}}+g\left(z_{i}\right) \sum_{i=1}^{N_{b}} e^{i k_{0} z_{i}}+X g\left(z_{j}\right) \sum_{j=1}^{N_{i}} e^{i k_{0} z_{j}}}{M} ; \\
& \int_{0}^{1} G_{\tau}\left(z-z_{i}\right) e^{i k_{0} z} d z=e^{i k_{0} z_{i}} \int_{-z_{i}}^{\lambda_{i} z_{i}} G_{\tau}(z) e^{i k_{z} z} d z=e^{i k_{0} z_{i}} g\left(z_{i}\right) \\
& g\left(z_{i}\right)=\int_{-z_{i}}^{\lambda_{0}-z_{i}} G_{\tau}(z) e^{i k_{0} z} d z
\end{aligned}
$$

where $\mathrm{M}$ is number of electron in a wavelet $\left\{0, \lambda_{o} \equiv 2 \pi / k_{o}\right\}$.

Let's calculate a RMS value of the bunching factor, assuming absence of correlation between electrons and hadrons, i.e. assuming a random Poisson distribution of their initial phases:

$$
\begin{aligned}
& \left\langle|M b(\tau)|^{2}\right\rangle=\left|\sum_{i, z_{i} \in\left\{0, \lambda_{0}\right\}} e^{i k_{0} z_{i}}+g\left(z_{i}\right) \sum_{i=1}^{N_{e}} e^{i k_{0} z_{i}}+X\left(z_{j}\right) g\left(z_{j}\right) \sum_{i=1}^{N_{j}} e^{i k_{k_{j}} z_{i}}+\right|^{2} \\
& \left|\sum_{i, z_{i} \in\left\{0, \lambda_{0}\right\}} e^{i k_{0} z_{i}}+g\left(z_{i}\right) \sum_{i=1}^{N} e^{i k_{0} z_{i}}\right|^{2}=\sum_{i, z_{i} \in\left\{0, \lambda_{b}\right\}}\left(1+2 \operatorname{Re} g\left(z_{i}\right)\right)+\sum_{i=1}^{N_{e}}\left|g\left(z_{i}\right)\right|^{2}+X^{2} \sum_{j}^{N_{i}}\left|g\left(z_{j}\right)\right|^{2}
\end{aligned}
$$

In other words, the

$$
\begin{aligned}
& \left\langle|M b(\tau)|^{2}\right\rangle=M+2 \sum_{i, z_{i} \in\left\{0, \lambda_{0}\right\}} \operatorname{Re} g\left(z_{i}\right)+\sum_{i=1}^{N_{e}}\left|g\left(z_{i}\right)\right|^{2}+\sum_{j}^{N_{i}} X^{2}\left(z_{j}\right)\left|g\left(z_{j}\right)\right|^{2} \\
& \left\langle\mid M b(\tau)^{2}\right\rangle=M\left(1+2 \cdot\langle\operatorname{Re} g(z)\rangle_{z \in\left\{0, \lambda_{0}\right\}}\right)+\int_{-\infty}^{\infty} \Lambda_{e}(z)|g(z)|^{2} d z+\int_{-\infty}^{0} \Lambda_{I}(z) X^{2}(z)|g(z)|^{2} d z
\end{aligned}
$$

where $\Lambda_{e}$ and $\Lambda_{i}$ are linear density of electrons and ions:

${ }^{1}$ To be exact, we should use a convolution of the induced density modulation in the modulator with Green function:

$$
X \sum_{j=1}^{N_{i}} G_{\tau}\left(z-z_{j}\right) \rightarrow \sum_{j=1}^{N_{i}} G_{\tau h}\left(z-z_{j}\right) ; \quad G_{\tau h}\left(z-z_{j}\right)=\int G_{\tau h}(z-\zeta) \rho_{h}\left(\zeta-z_{j}\right) d \zeta .
$$

where $\rho_{h}$ is a density modulation induced by the hadron in the CeC modulator [1]. For cases of interest, the details of $\rho_{h}$ are not important, when its duration is much shorter than that of the amplifier. 


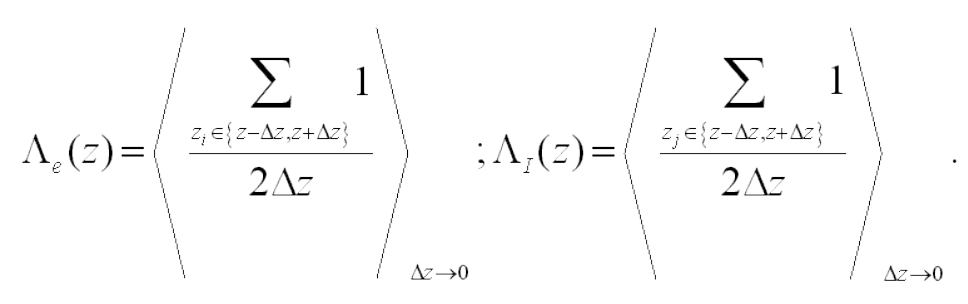

For simplicity let's look at the contentious beam with fixed density:

Then

$$
\Lambda_{e}=\frac{M_{e}}{\lambda_{0}} ; \Lambda_{I}=\frac{M_{I}}{\lambda_{0}} \text {. }
$$

$$
\left\langle|M b(\tau)|^{2}\right\rangle=M\left(1+2 \cdot\langle\operatorname{Reg}(z)\rangle_{z \in\left\{0, \lambda_{0}\right\}}\right)+\Lambda_{e} \int_{-\infty}^{\infty}|g(z)|^{2} d z+X^{2} \cdot \Lambda_{I} \int_{-\infty}^{\infty}|g(z)|^{2} d z \text { (8) }
$$

Causality in FEL means that the $|g(z)|_{z \in\left\{0, b_{0}\right\}} \rightarrow 0$. Thus, the modulation is determined by the effective correlation length, which is defined as [1]:

$$
\int_{-\infty}^{\infty}|g(z)|^{2} d z=g_{\max }^{2} N_{c} \lambda_{0}
$$

Then, assuming that the FEL is saturated at $\mathrm{b}<1$;

$$
1+g_{\max }^{2} N_{c}\left(1+X^{2} \cdot \frac{M_{I}}{M_{e}}\right) \leq M_{e}
$$

gives an estimate for the maximum attainable gain for an initial $\delta$-function:

$$
g_{\max } \leq \sqrt{\frac{M}{N_{c}\left(1+X^{2} \cdot \frac{M_{I}}{M_{e}}\right)}}
$$

To be exact, for am FEL operating at peak current of $\mathrm{I}_{\mathrm{p}}$ and wavelength $\lambda_{o}$,

$$
M_{e}=\frac{I_{p e} \lambda_{o}}{e c}=2.08 \cdot 10^{4} \cdot I_{p}[A] \lambda_{o}[\mu m]
$$

This gives a following limitation on the maximum attainable gain:

$$
g_{\max } \leq 144 \cdot \sqrt{\frac{I_{p e}[A] \cdot \lambda_{0}[\mu m]}{N_{c}\left(1+\frac{X^{2}}{Z} \cdot \frac{I_{p I I}}{I_{p e}}\right)}}
$$

Since from plasma oscillations in a modulator [1], $X \leq 2 Z$ 


$$
g_{\max } \sim 144 \cdot \sqrt{\frac{I_{p e}[A] \cdot \lambda_{o}[\mu m]}{N_{0}\left(1+Z^{*} \frac{I_{p H I}}{I_{p e}}\right)}} ; Z^{*}=X^{2} / Z
$$

and

$$
g_{\max } \sim \sqrt{\frac{I_{p e} \lambda_{o}}{e c \cdot N_{c}\left(1+Z^{*} \frac{I_{p I I}}{I_{p e}}\right)}}
$$

To move further we can express the FEL wavelength through other parameters:

$$
\lambda_{0}=\frac{\lambda_{w}\left(1+a_{w}^{2}\right)}{2 \gamma^{2}}
$$

transferring it to

$$
g_{\max } \sim \sqrt{\frac{I_{p e} \lambda_{w}\left(1+a_{w}^{2}\right)}{2 \gamma^{2} e c \cdot N_{c}\left(1+Z^{*} \frac{I_{\tilde{B} I}}{I_{p e}}\right)}}
$$

This result does not depend neither on the type of FEL nor on the properties of the electron beam (assuming it is suitable for FEL) and fully applicable for 3D treatment. At the same time there is no analytical expression for $N_{c}$ for an arbitrary 3D FEL.

\section{D FEL case}

Hence, to move further, we need to make an approximation for $N_{c}$ using a simple 1D theory. Here the expression derived in [2] :

$$
N_{c} \propto \sqrt{\frac{N_{w}}{9 \sqrt{3} \rho}}
$$

giving

$$
g_{\max } \sim \sqrt{\frac{I_{p e} \lambda_{w}\left(1+a_{w}^{2}\right)}{2 \gamma^{2} e c \cdot \sqrt{\frac{N_{w}}{9 \sqrt{3} \rho}}\left(1+Z^{*} \frac{I_{p I}}{I_{p e}}\right)}}
$$

Using 1D definition

$$
L_{G}=\frac{1}{2 \sqrt{3} k_{w} \rho}
$$


and

$$
\begin{aligned}
& N_{w} \equiv \frac{L_{w}}{\lambda_{w}}=\operatorname{Ln} n_{F E L} \frac{L_{G}}{\lambda_{w}}=\operatorname{Ln} n_{F E L} \cdot \frac{1}{4 \pi \sqrt{3} \rho} \\
& \operatorname{Ln}_{F E L} \sim 18-20
\end{aligned}
$$

where $L n_{F E L}$ number of the gain lengths needed for FEL to saturate.

$$
g_{\max } \sim \sqrt{\frac{3 \sqrt{3 \pi} \cdot I_{p e} \lambda_{w}\left(1+a_{w}^{2}\right) \rho}{\gamma^{2} e c \cdot \sqrt{L n_{F E L}}\left(1+Z^{*} \frac{I_{p I I}}{I_{p e}}\right)}}
$$

Finally, in 1D case we shell conclude that maximum gain attainable for $\mathrm{CeC}$ is:

$$
g_{\max } \sim \sqrt{\frac{3 \sqrt{3 \pi} \cdot I_{p e} \lambda_{w}\left(1+a_{w}^{2}\right)}{2 \gamma^{2} e c \cdot \sqrt{L n_{F E L}}\left(1+Z^{*} \frac{I_{p I I}}{I_{p e}}\right)} \sqrt[3]{\frac{2 \lambda_{w} I_{e} e}{\gamma m c^{3} k_{o} S} \frac{a_{w}^{2}}{1+a_{w}^{2}}}}
$$

\section{References}

[1] V.N. Litvinenko, Ya.S. Derbenev, Physical Review Letters 102, 114801 (2009)

[2] S. Krinsky, AIP Conference Proceedings 648, 23 (2002) 